\section{Original Article}

Microbiology

Check for updates

\title{
Annexin A2 gene interacting with viral matrix protein to promote bovine ephemeral fever virus release
}

\author{
Lihui Chen (D), Xingyu Li $\mathbb{D}$, Hongmei Wang (D) ", Peili Hou (D) *, Hongbin He ${ }^{(D)}$ * \\ Ruminant Diseases Research Center, College of Life Sciences, Shandong Normal University, Jinan 250014, \\ China
}

Received: Sep 3, 2019

Revised: Nov 11, 2019

Accepted: Dec 30, 2019

*Corresponding authors:

Hongmei Wang

Ruminant Diseases Research Center, College

of Life Sciences, Shandong Normal University,

88 Wenhua E Rd, Lixia, Jinan 250014, China.

E-mail: hongmeiwang@sdnu.edu.cn

Peili Hou

Ruminant Diseases Research Center, College of Life Sciences, Shandong Normal University, 88 Wenhua E Rd, Lixia, Jinan 250014, China.

E-mail: apeilihou@163.com

\section{Hongbin $\mathrm{He}$}

Ruminant Diseases Research Center, College of Life Sciences, Shandong Normal University, 88 Wenhua E Rd, Lixia, Jinan 250014, China. E-mail: hongbinhe@sdnu.edu.cn

(c) 2020 The Korean Society of Veterinary Science

This is an Open Access article distributed under the terms of the Creative Commons Attribution Non-Commercial License (https:// creativecommons.org/licenses/by-nc/4.0) which permits unrestricted non-commercial use, distribution, and reproduction in any medium, provided the original work is properly cited.

ORCID iDs

Lihui Chen (iD

https://orcid.org/0000-0002-1492-5704 Xingyu Li (iD)

https://orcid.org/0000-0002-4242-3023 Hongmei Wang (D)

https://orcid.org/0000-0002-4094-6904

\begin{abstract}
Bovine ephemeral fever virus (BEFV) causes bovine ephemeral fever, which can produce considerable economic damage to the cattle industry. However, there is limited experimental evidence regarding the underlying mechanisms of BEFV. Annexin A2 (AnxA2) is a calcium and lipid-conjugated protein that binds phospholipids and the cytoskeleton in a $\mathrm{Ca}^{2+}-$ dependent manner, and it participates in various cellular functions, including vesicular trafficking, organization of membrane domains, and virus proliferation. The role of the AnxA2 gene during virus infection has not yet been reported. In this study, we observed that AnxA2 gene expression was up-regulated in BHK-21 cells infected with the virus. Additionally, overexpression of the AnxA2 gene promoted the release of mature virus particles, whereas BEFV replication was remarkably inhibited after reducing AnxA2 gene expression by using the small interfering RNA (siRNA). For viral proteins, overexpression of the Matrix (M) gene promotes the release of mature virus particles. Moreover, the AnxA2 protein interaction with the M protein of BEFV was confirmed by GST pull-down and coimmunoprecipitation assays. Experimental results indicate that the C-terminal domain (268$334 \mathrm{aa})$ of AxnA2 contributes to this interaction. An additional mechanistic study showed that AnxA2 protein interacts with $M$ protein and mediates the localization of the $M$ protein at the plasma membrane. Furthermore, the absence of the AnxA2-V domain could attenuate the effect of AnxA2 on BEFV replication. These findings can contribute to elucidating the regulation of BEFV replication and may have implications for antiviral strategy development.
\end{abstract}

Keywords: Bovine ephemeral fever virus; annexin A2; matrix protein; viral replication

\section{INTRODUCTION}

Bovine ephemeral fever, also known as bovine influenza, was first recorded in the late 19th century $[1,2]$. It can produce acute febrile illness in cattle and water buffalo, leading to economic losses through the associated acute high fever, muscle stiffness, and a decrease in milk production $[3,4]$. Its causative agent is the bovine ephemeral fever virus (BEFV), which is a member of the genus Ephemerovirus in the family Rhabdoviridae. BEFV is an enveloped, negative-sense, single-chain RNA virus [5]. The viral genome consists of five constitutive proteins contained as $3^{\prime}-\mathrm{N}-\mathrm{P}-\mathrm{M}-\mathrm{G}-\mathrm{L}-5^{\prime}$, as well as six non-structural proteins: $\mathrm{G}_{\mathrm{NS}}, \alpha 1, \alpha 2, \alpha 3$, 
Peili Hou (iD

https://orcid.org/0000-0002-4750-6952

Hongbin $\mathrm{He}$ (iD)

https://orcid.org/0000-0002-7438-0638

Funding

This study was partially supported by grants from the National Natural Science Fund of China (31872490, 31672556), the Primary

Research \& Development Plan of Shandong Province (2018GNC113011), and a fund earmarked for the Taishan Scholar Project (Hongbin $\mathrm{He}$ ).

Conflict of Interest

The authors declare no conflicts of interest.

Author Contributions

Formal analysis: Chen L, Hou P, Li X; Funding acquisition: $\mathrm{He} \mathrm{H}$, Wang $\mathrm{H}$; Project administration: $\mathrm{He} \mathrm{H}$, Hou P, Wang $\mathrm{H}$; Writing - original draft: Chen L, Hou P; Writing - review \& editing: Chen L, Hou P, Li X. $\beta, \gamma[1,6]$. Among those viral proteins, the matrix (M) protein has a mass of about $27 \mathrm{kDa}$ and consists of 224 amino acids. It is a lipid bi-layer inner membrane protein encoded by the $\mathrm{M}$ gene and is distributed between viral the envelope and the nuclear capsid. It is an important component of the viral particles of the Rhabdoviridae, and the functions of M proteins of other viruses have been studied in rabies virus (RABV) [7] and vesicular stomatitis virus (VSV) [8]. Nevertheless, there are no previous reports on the function of the M protein of BEFV.

Annexins are calcium or phospholipid-binding molecules that exist mainly in eukaryotes $[9,10]$. Annexin A2 (AnxA2), also called calpectin 1 or Annexin II, is a member of the Annexin family [11,12] that is involved in endocytosis, exocytosis [13], the organization of membrane domains, extracellular receptor activity [14], vesicular trafficking [15], cell proliferation [16,17], protein assembly $[12,18]$, and tumor cell invasion. AnxA2 is has a mass of approximately $40 \mathrm{kDa}$ and is comprised of 340 amino acids. Like other members of the Annexin family, AnxA2 has a volatile $\mathrm{N}$-terminal domain and a conserved $\mathrm{C}$-terminal protein core [9]. Furthermore, the $\mathrm{N}$-terminal domain includes an S100A10 site and phosphorylation sites $[19,20]$. The C-terminal contains four highly homologous domains (named II-V) of 70-80 amino acids, which together produce a closely packed bending structure with concave and convex edges [21]. AnxA2 is reported to be associated with mRNAs at the $\mathrm{C}$-terminal $[22,23]$ and comprises a combining site for F-actin, plasminogen, and heparin [12]. Recently, AnxA2 has been reported to be associated with the life cycles of multiple viruses including cytomegalovirus (CMV) [24,25], hepatitis C virus (HCV) [26], influenza A virus (IAV) [27,28], and others [29,30]. However, the function of the AnxA2 gene in the life cycle of Rhabdoviridae viruses has not been described.

In this study, we investigated whether endogenous AnxA2 gene expression was affected during BEFV infection and whether the AnxA2 gene had a significant effect on virus replication. In addition, the role of the BEFV M gene on virus proliferation was explored. Also, the relationship between the AnxA2 and M proteins was examined to determine whether there is an interaction between the two proteins and determine the specific domain of such interaction. Furthermore, the mechanism of AnxA2 protein promotion of viral proliferation in terms of the membrane localization of $\mathrm{M}$ protein was preliminarily studied.

\section{MATERIALS AND METHODS}

\section{Cells and virus}

Baby hamster kidney (BHK-21) cells and human embryonic kidney 293 (HEK-293T) cells were grown in Dulbecco's modified Eagle medium (DMEM, Gibco, USA) containing 10\% fetal bovine serum (FBS, Gibco), $100 \mathrm{U} / \mathrm{mL}$ penicillin $\mathrm{G}$, and $100 \mathrm{mg} / \mathrm{mL}$ streptomycin sulfate (Gibco). The cells were cultured in an atmosphere containing $5 \% \mathrm{CO}_{2}$ at $37^{\circ} \mathrm{C}$. At $80 \%-90 \%$ confluency, BEFV (Shandong/China/2011) were infected into BHK-21 cells at a multiplicity of infection (MOI) of 0.1 and fostered in DMEM containing $2 \%$ FBS.

\section{Plasmid construction}

A recombinant plasmid including the $M$ gene with the HA-tag sequence was constructed using a pcDNA3.1(+) vector. Briefly, the full-length $\mathrm{M}$ gene of BEFV was amplified by RT-PCR and RNA of BEFV was extracted by using Trizol reagent (Invitrogen, USA). Finally, the M gene was constructed into the pcDNA3.1-HA vector with the HA-tag sequence at the $\mathrm{N}$-terminal and the construct was named pcDNA3.1-HA-M. 
Recombinant plasmids including the AnxA2 gene and its mutants with the Flag-tag sequence were constructed using the pcDNA3.1 and pLVX-IRES-Puro vectors, respectively. The total cellular RNA was obtained from BHK-21 cells using Trizol reagent (Invitrogen). The Thermo Scientific RevertAid First Strand cDNA Synthesis kit (Thermo Scientific, USA) was used for reverse transcription [31]. The AnxA2 gene was amplified with AnxA2 primers including the Flag-tag sequence at the C-terminus (Table 1). The AnxA2 fragment was then constructed into the pcDNA3.1 and pLVX-IRES-Puro vectors, and the recombinant plasmids obtained were named pcDNA3.1-AnxA2-Flag and pLVX-AnxA2-Flag-IRES-Puro, respectively. According to previous descriptions [32,33], the structural domains of AnxA2 from the $\mathrm{N}$-terminal to the C-terminal were as follows (Table 1): AnxA2-I (1-36 aa), AnxA2-II (37-102 aa), AnxA2-III (108-174 aa), AnxA2-IV (192-259 aa), and AnxA2-V (268-334 aa). Accordingly, five AnxA2 mutant plasmids of expressing the Flag-tag sequence and lacking the corresponding domains were constructed into pcDNA3.1 and named AnxA2- $\Delta \mathrm{I}$, AnxA2- $\Delta \mathrm{II}, \mathrm{AnxA2}-\Delta \mathrm{III}, \mathrm{AnxA2}-\Delta \mathrm{IV}$, and $A n x A 2-\Delta \mathrm{V}$, respectively. In addition, the $\mathrm{C}$-terminal domain of AnxA2 was divided into AnxA2-C1 (37-186 aa) and AnxA2-C2 (187-340 aa). Two AnxA2 mutant plasmids expressing the Flag-tag sequence were cloned into pcDNA3.1 and named AnxA2-C1 and AnxA2-C2, respectively. Finally, the recombinant plasmids were confirmed by DNA sequencing. After the recombinant vectors were successfully constructed, the expression of proteins was assessed following transfection of HEK-293T cells.

\section{Detection of AnXA2 mRNA levels by real-time qPCR}

The AnxA2 mRNA levels were examined by performing real-time qPCR as described previously [34]. Briefly, BHK-21 cells were infected with BEFV at an MOI of 0.1, and the cells were collected after $6 \mathrm{~h}, 12 \mathrm{~h}, 24 \mathrm{~h}$, and $48 \mathrm{~h}$ post-infection (hpi). Cellular RNA was extracted from cell samples by using the RNA prep Pure Cell kit (Qiagen, USA) [35]. The total RNA was reversed transcribed using the Prime Script RT reagent kit (TaKaRa, Japan). SYBR green-based real-time qPCR was conducted using a Premix Ex Taq kit (Japan). The expression of AnxA2 mRNA was detected by a Roche Light Cycler 480 Real-Time PCR System (Roche Applied Science, Germany). The threshold cycle (Ct) value associated with the RNA levels of each objective gene was standardized to the GAPDH expression level, and the relative expression of each specimen was analyzed by applying the previously described $2^{-\Delta \Delta \mathrm{Ct}}$ method [36].

\section{Analysis of AnxA2 protein expression by western blotting}

The infection of BHK-21 cells with BEFV was performed at an MOI of 0.1, and cell samples were collected at 12, 24, 36, and $48 \mathrm{hpi}$. The obtained samples were subjected to $12.5 \%$

Table 1. List of primers used to create AnxA2-WT and AnxA2 deletion mutants

\begin{tabular}{|c|c|c|}
\hline Primer name & Sequence $\left(5^{\prime}-3^{\prime}\right)$ & Application \\
\hline AnxA2-WT-F & CGCGGATCCGCCACCATGTCTACCGTTCATGAAATTC & \multirow{2}{*}{$\begin{array}{c}\text { Construct recombinant plasmids of } \\
\text { pLVX-IRES-Puro }\end{array}$} \\
\hline AnxA2-WT-R & CCGGAATTCTCACTTGTCATCGTCGTCCTTGTAATCGTCATCCCCACCACACAGG & \\
\hline AnxA2- $\Delta \mathrm{I}-\mathrm{F}$ & CGCGGATCCGCCACCATGCGGGATGCTCTGAACATTG & \multirow{10}{*}{$\begin{array}{l}\text { Construct recombinant plasmids of } \\
\qquad \operatorname{pcDNA3.1(+)}\end{array}$} \\
\hline AnXA2- $\Delta I-R$ & CCGGAATTCTCACTTGTCATCGTCGTCCTTGTAATCGTCATCCCCACCACACAGG & \\
\hline AnxA2- $\Delta I I-R$ & AGCAGGTGTTTTCAACTCAGCATCAAAGTTAGTG & \\
\hline AnxA2- $\Delta I I-F$ & AACTTTGATGCTGAGTTGAAAACACCTGCTCAGT & \\
\hline AnxA2- $\triangle \mathrm{III}-\mathrm{F}$ & AAAACACCTGCTCAGGCAAAGGGTCGGAGAGCAG & \\
\hline AnxA2- $\Delta$ III-R & TCTCCGACCCTTTGCCTGAGCAGGTGTTTTCAAT & \\
\hline AnXA2- $\Delta \mathrm{IV}-\mathrm{F}$ & TATGAACTGATTGACGTTCAGTGCATTCAGAACA & \\
\hline AnxA2- $\Delta I V-R$ & CTGAATGCACTGAACGTCAATCAGTTCATAATCA & \\
\hline AnxA2- $\Delta V-F$ & CGCGGATCCGCCACCATGTCTACCGTTCATGAAATTC & \\
\hline AnxA2- $\Delta V-R$ & CCGGAATTCTCACTTGTCATCGTCGTCCTTGTAATCGTCATCCCCACCACACAGGGGCTTGTTCTGAATG & \\
\hline
\end{tabular}

Bold letters indicate the restriction endonuclease enzyme cutting sites; Underlined letters indicate the Flag-Tag sequence. AnxA2, annexin A2; WT, wild type; F, forward; R, reverse. 
SDS gel electrophoresis and the gel products shifted to PVDF membranes. The spots were sealed with TBST containing 8\% skim milk powder. Anti-AnxA2 antibody (Santa Cruz Biotechnology) was diluted by 1:2,000, and anti- $\beta$-actin antibody (Abways, China) was used at a fluxing dilution of 1:10,000. In accordance with the manufacturer's agreement, an enhanced chemiluminescence reagent was used to visualize the membranes.

\section{Establishing of stable-expression AnxA2 gene cell lines}

To establish cell lines that stably express AnxA2, the AnxA2 recombinant plasmid pLVXAnxA2-IRES-Puro was transfected into BHK-21 cells as previously described [37]. The pLVX-IRES-Puro plasmid was transfected into BHK-21 cells as the control. The treated cells were planted in fresh medium and sifted for puromycin $(2-5 \mu \mathrm{g} / \mathrm{mL})$ every $3-4$ days until monoclonal colonies were acquired. Subsequently, monoclonal puromycin-resistant colonies were selected, AnxA2 gene expression in the two cell lines was tested by western blot analysis using anti-Flag rabbit antibody (Cell Signaling Technology, USA) and anti-AnxA2 mouse antibody, respectively (Santa Cruz Biotechnology, USA). An AnxA2-overexpressing cell line (BHK-AnxA2) and a control cell line (BHK-NC) were successfully constructed [38].

\section{Small interfering RNA (siRNA) analysis}

In order to reduce endogenous AnxA2 gene expression in BHK-21 cells, the siRNAs targeting the AnxA2 gene and the negative control sequences were designed and compounded by Biomics Biotechnology (China). The siRNA sequences with the deoxythymidine dinucleotide (dTdT) overhangs are summarized in Table 2. Effective siRNA-AnxA2 targeting the AnxA2 gene (siAnxA2) and scrambled siRNA used as a control of AnxA2 siRNA were transfected into BHK-21 cells at the $50 \mathrm{nM}$ level using Attractene Transfection Reagent (Qiagen, Germany) until obtaining a confluence of $80 \%$ in 6-well plates, respectively. The knock-down efficiency of the AnxA2 gene based on siRNA analysis was confirmed by western blot analysis.

\section{Co-immunoprecipitation (Co-IP) assay}

BHK-21 cells were placed in $10 \mathrm{~cm}$ plates and transfected with pcDNA3.1-HA-M and pcDNA3.1-AnxA2-Flag or pcDNA3.1-HA-M and pcDNA3.1-Flag. In addition, BHK-21 were transfected with pcDNA3.1-HA-M and the corresponding recombined plasmids expressing the AnxA2 mutants. Prior to performing Co-IP assays, cell samples were collected $24 \mathrm{~h}$ posttransfection (hpt) and washed three times with phosphate buffer saline (PBS). The Co-IP assays were performed using an immunoprecipitation kit (Thermo-Fisher, USA) according to a previously described procedure [33]. Finally, after lysis buffer splitting of the samples, the protein samples were detected by western blotting. The obtained membranes were combined with anti-Flag rabbit antibody (Cell Signaling Technology, USA) or anti-HA antibody (Cell Signaling Technology).

Table 2. List of primers used during knock-down of the AnxA2 gene

\begin{tabular}{lll}
\hline Primer name & \multicolumn{1}{c}{ Sequence $\left(5^{\prime}-3^{\prime}\right)$} & Application \\
\hline AnxA2-siRNA-1 & CCCUGUACUACUAUAUUCAdTdT & \\
AnxA2-siRNA-2 & UGAUAUAGUAGUACAGGGdTdT & \\
& UACAUUUCCUUGUAGACUCdTdT & \\
AnxA2-siRNA-3 & GUCAAAGCGUACACUAACUdTdT & \\
AnxA2-siRNA-4 & AGUUAGUGUACGCUUUGACdTdT & Knock-down of AnxA2 gene \\
SiNC & GUGAAGUGGACAUGUUGAAdTdT & \\
& UUCAACAUGUCCACUUCACdTdT & \\
& UUCUCCGAACGUGUCACGUdTdT & \\
\hline
\end{tabular}

AnxA2, annexin A2; siRNA, small interfering RNA; siNC, scrambled siRNA used as a negative control for AnxA2 siRNA. 


\section{GST pull-down assay}

Optimized fusion expression of the M protein has been confirmed in our laboratory. Initially, the GST fusion protein (pGEX-4T-1-M) was expressed in Escherichia coli BL21 cells by culturing them at $37^{\circ} \mathrm{C}$ in medium containing $100 \mathrm{mg} / \mathrm{L}$ ampicillin. When the $\mathrm{OD} 600$ reached 0.6 , $1 \mathrm{mM} \mathrm{IPTG}$ was added and the cells held at $25^{\circ} \mathrm{C}$ overnight with shaking. Sediments were collected after centrifugal separation at $4^{\circ} \mathrm{C}$. Precipitation was suspended by using a lysis buffer followed by sonication. The GST-M purity was assessed by performing sodium dodecyl sulfate polyacrylamide gel electrophoresis (SDS-PAGE).

The recombinant plasmid pcDNA3.1-AnxA2-Flag was transfected into BHK-21 cells for $24 \mathrm{~h}$ and collected in centrifuge tubes, and add lysate. The lysis supernatant was incubated with beads of purified GST-M overnight at $4^{\circ} \mathrm{C}$ with gentle shaking. After washing three times with PBS, the final protein samples were separated from the GST-M beads and boiled in $5 \times$ SDS loading buffer. Subsequently, the samples along with rabbit anti-Flag antibody (Cell Signaling Technology, USA) underwent western blot analysis.

\section{Plasma membrane separation assay}

BHK-21 cells were transfected with vector (pcDNA3.1) alone or pcDNA3.1-HA-M and pcDNA3.1 or pcDNA3.1-HA-M and pcDNA3.1-AnxA2-Flag, and at $24 \mathrm{hpt}$, transfected cells were collected and washed with PBS. Samples were harvested via low-temperature lowspeed centrifugation and were cleaned by using cold PBS. The cytoplasmic and plasma membrane (PM) protein components were separated and collected by using the Minute ${ }^{\mathrm{TM}}$ Plasma Membrane Protein Isolation and Cell Fractionation kit (Invent Biotechnologies, USA) [39]. The amounts of protein in the cytoplasm and the PM were determined by western blot analysis with the addition of $\beta$-actin (Abways) and $\mathrm{Na} / \mathrm{K}$ ATPase (Abways) antibodies.

\section{Statistical analyses}

Data were analyzed by using the tests within Graph Pad Prism software (version 8.0; USA). Statistical significance was estimated by using two-way variance analysis and multiple comparison tests. Multiple samples were assessed by performing one-way analysis of variance. The results are expressed as average values \pm SD obtained from at least three independent experiments. A $p$ value lower than 0.05 was considered statistically significant, and a $p$ value below 0.01 was considered highly significant.

\section{RESULTS}

\section{BEFV infection up-regulates AnxA2 gene expression in BHK-21 cells}

The AnxA2 gene's involvement in infections by some viruses has been previously reported. For the current investigation into whether BEFV infection influences AnxA2 gene expression, the AnxA2 gene expression level was examined in BHK-21 cells during BEFV infection. BHK21 cells infected with BEFV or mock-infected (as a control) were collected at 12, 24, and 48 hpi, and total RNA was extracted. Subsequently, the mRNA of the AnxA2 gene was detected by performing real-time qPCR. As shown in Fig. 1A, the AnxA2 mRNA level increased at different times compared to those of the control cells, especially at 48 hpi thus indicating that the AnxA2 mRNA level increased after virus infection. To further examine the expression of AnxA2 protein in BHK-21 cells, BEFV-infected BHK-21 cells were harvested at 12, 24, 36, and 48 hpi. AnxA2 proteins underwent western blot analysis and the results showed that AnxA2 
A

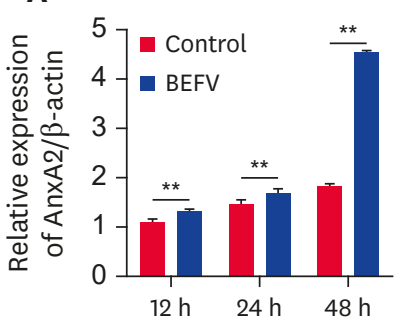

B

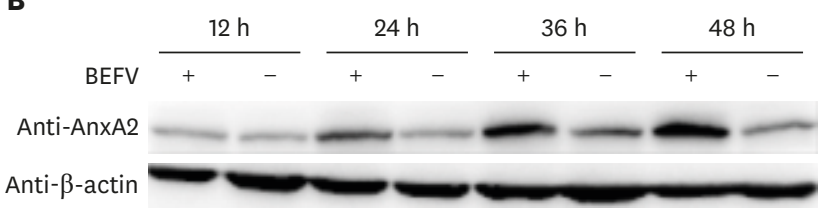

Fig. 1. BEFV infection up-regulates AnxA2 gene expression in BHK-21 cells. (A) AnxA2 gene expression levels in BHK-21 cells infected with BEFV at an MOI of 0.1 at 12, 24, and 48 hpi were determined by performing real-time qPCR with $\beta$-actin used as the standard. Data indicate the relative fold-change from the control cells. The average values $\pm S D$ were determined from three independent experiments. (B) AnxA2 protein expression in BHK-21 cells mock-infected or infected with BEFV at an MOI of 0.1 at 12, 24, 36, and 48 hpi determined by western blotting with anti-AnxA2 antibody and $\beta$-actin used as the control.

AnxA2, annexin A2; BEFV, bovine ephemeral fever virus; MOI, multiplicity of infection; hpi, hours post infection; qPCR, quantitative polymerase chain reaction. ${ }^{* *} p<0.01$.

protein expression was increased at 24, 36, and 48 hpi (Fig. 1B). In conclusion, the results suggest that BEFV infection increases the expression of the AnxA2 gene in BHK-21 cells.

\section{AnxA2 gene promotes BEFV replication in BHK-21 cells}

To study the role of the AnxA2 gene in BEFV replication, cell lines that stably overexpressed the AnxA2 gene (BHK-AnxA2) were established. In addition, the corresponding negative control cell lines (BHK-NC) were successfully constructed. As shown in Fig. 2A, expression of AnxA2 protein was successfully detected by western blot analysis using anti-Flag and anti-AnxA2 antibodies. The BHK-AnxA2 and BHK-NC cell lines were infected with BEFV and cell supernatants collected at $48 \mathrm{hpi}$. The results showed that the virus titer obtained in the BHK-AnxA2 cell supernatant was 3-fold higher than that in the BHK-NC control cells (Fig. 2B). Therefore, the results show that overexpression of the AnxA2 gene may promote virus replication.

To further investigate the role of the AnxA2 gene on BEFV growth, AnxA2-specific siRNA (siAnxA2) and control siRNA (siNC) were transfected into BHK-21 cells (Table 2). As shown in Fig. 2C, siAnxA2 significantly inhibited AnxA2 gene expression compared to that of the control siRNA. Accordingly, siAnxA2 and siNC cells were infected with BEFV and cell supernatants were gathered at 48 hpi. Virus titers in the cell supernatant of siAnxA2-infected cells were markedly lower than those in the siNC cells (Fig. 2D). These results show that knock-down of expression of AnxA2 markedly decreases virus yield.

In short, these results indicate that the AnxA2 gene can, as a cellular factor, promote the production of BEFV particle progeny.

\section{BEFV M gene promotes virus replication in BHK-21 cells}

It has been reported that the VSV M protein can promote virus release [8]. However, it is unclear whether the BEFV M protein can promote the release of the virus. The pcDNA3.1HA-M and control vectors were separately transfected into BHK-21 cells. At $24 \mathrm{hpt}$, expression of the $\mathrm{M}$ protein was detected by western blot analysis (Fig. 3A). The vector-transfected cells were then infected with BEFV. Cell supernatants and total cell precipitations were collected at $48 \mathrm{hpi}$. Interestingly, compared with the vector control, the virus titer of the transfected pcDNA3.1-HA-M cells in the cell supernatant was significantly increased (Fig. 3B), whereas, the virus titers of the total cell precipitates exhibited no significant change (Fig. $\mathbf{3 C}$ ). 
A

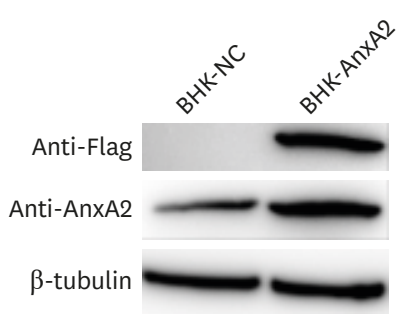

C

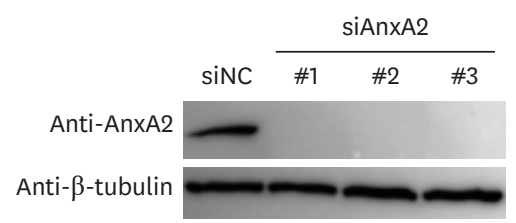

B

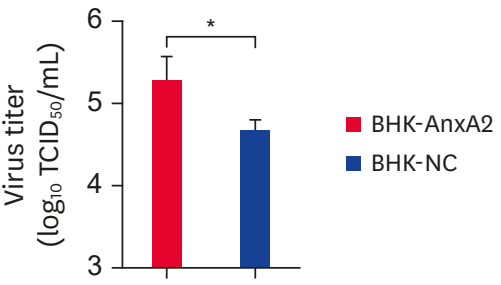

D

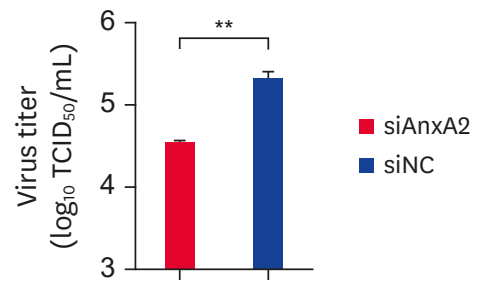

Fig. 2. AnxA2 gene expression promotes BEFV replication in BHK-21 cells. (A) The expression levels of AnxA2 protein in a stable AnxA2 overexpression cell line (BHK-AnxA2) and the negative control cell line (BHK-NC) were assayed by western blotting with anti-AnxA2 and anti-Flag antibody; $\beta$-actin was used as the control. (B) BHK-AnXA2 and BHK-NC cells were inoculated with BEFV at an MOI of $0.1 \mathrm{for} 48 \mathrm{~h}$, and the resulting titers in the supernatant of BEFV-infected cells were determined by $\mathrm{TCID}_{50}$ assay. The mean and standard deviation values were determined from three independent experiments. (C) The knock-down efficiency of AnxA2-siRNA in BHK-21 cell lines was examined by western blot analysis with anti-AnxA2 antibody and control siRNA with $\beta$-actin as the internal control. (D) SiAnXA2 and siNC were infected with BEFV at an MOI of $0.1 \mathrm{for} 48 \mathrm{~h}$ and the release of progeny virus particles in cell supernatants was assessed. The data are average and SD values obtained from three independent experiments.

AnxA2, annexin A2; BEFV, bovine ephemeral fever virus; MOI, multiplicity of infection; siRNA, small interfering RNA; siNC, scrambled siRNA for AnxA2; siAnxA2, effective siRNA-AnxA2 targeting the AnxA2 gene; NC, negative control; $\mathrm{TCID}_{50}$, median tissue culture infective dose.

${ }^{*} p<0.05 ;{ }^{* *} p<0.01$.

A

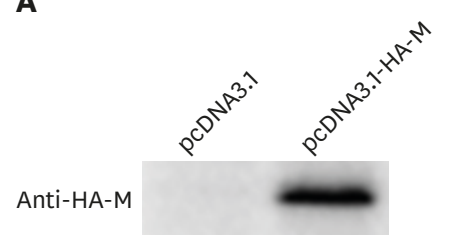

B

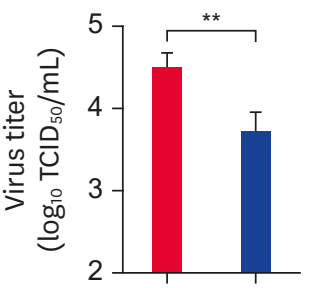

C

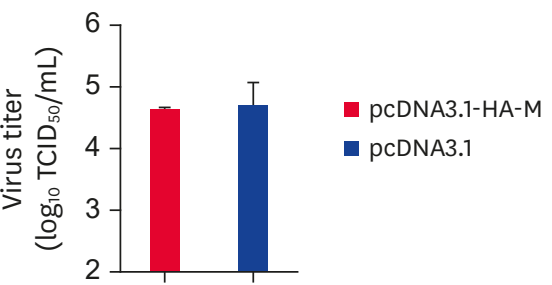

Fig. 3. The M gene promotes BEFV replication in BHK-21 cells. (A) pcDNA3.1-HA-M and the vector control were transfected separately into BHK-21 cells. At 24 hpt, the expression of $\mathrm{M}$ protein was assayed by western blotting with rabbit anti-HA. (B) BHK-21 cells were transfected with pcDNA3.1-HA-M and the vector control at $24 \mathrm{hpt}$ and infected with BEFV at an $\mathrm{MOI}$ of 0.1 . After $48 \mathrm{hpi}$, cell supernatants were collected and assayed by determining TCID $\mathrm{D}_{50}$. $(\mathrm{C})$ Cell precipitates were collected and assayed by determiningTCID ${ }_{50}$.

M, matrix; BEFV, bovine ephemeral fever virus; $M O I$, multiplicity of infection; hpi, hours post infection; $\mathrm{TCID}_{50}$, median tissue culture infective dose. ${ }^{* *} p<0.01$.

Therefore, it is surmised that M protein presence promotes BEFV proliferation, especially the release of mature virus particles.

\section{AnXA2 protein interacts with BEFV M protein in BHK-21 cells}

The above results show that BEFV infection up-regulates AnxA2 gene expression. In addition, both the AnxA2 and M proteins can promote BEFV replication in BHK-21 cells. Therefore, the relationship between the AnxA2 and M proteins was examined by performing GST pulldown assays. GST-M was purified, the AnxA2 gene was transfected into BHK-21 cells, and at $24 \mathrm{hpt}$, cells were collected and the cell pyrolysis product precipitated with GST-tagged fusion proteins (GST-M). The results showed that the AnxA2 protein does interact with the BEHV M protein (Fig. 4A). Subsequently, the interaction between AnxA2 and M proteins was further confirmed by performing Co-IP, in which pcDNA3.1-AnxA2-Flag and pcDNA3.1- 

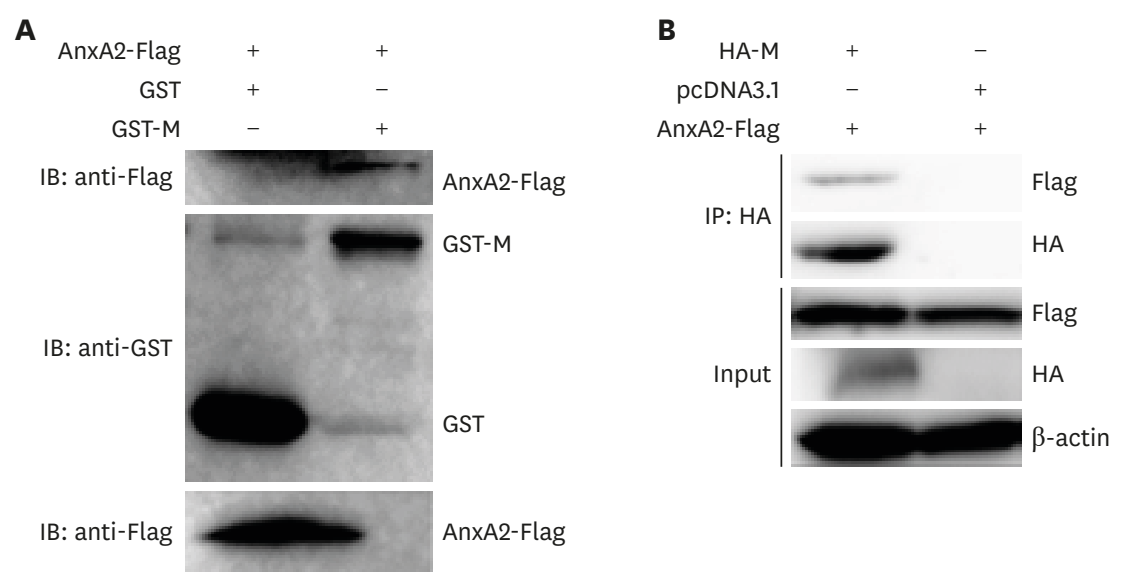

Fig. 4. AnxA2 interacts with bovine ephemeral fever virus $M$ protein in BHK-21 cells. (A) AnxA2 interaction with $M$ assessed by GST pull-down assay. GST or GST-M fusion protein was combined with BHK-21 cell lysates expressing pcDNA3.1-AnXA2-Flag. GST and GST-M were combined with Beaver Beads (GSH). After washing, proteins were eluted from the beads and detected by performing sodium dodecyl sulfate polyacrylamide gel electrophoresis. The presence of AnxA2 was tested by immune-blotting with rabbit anti-Flag. The expression of GST and GST-M was determined by immunoblotting with rabbit anti-GST. (B) The interaction between AnXA2 and M proteins was identified by performing co-immunoprecipitation assays. BHK-21 cells were co-transfected with pcDNA3.1-AnxA2-Flag and pcDNA3.1-HA-M. At $24 \mathrm{hpt}$, whole-cell lysates were immunoprecipitated with the anti-HA antibody. Subsequently, the complex underwent western blot analysis using specific antibodies (anti-HA and anti-Flag) as indicated.

AnxA2, annexin A2; M, matrix.

HA-M were co-transfected in BHK-21 cells. The Co-IP was performed with anti-HA, and the AnxA2 protein was detected in a co-transformation complex by using the anti-Flag antibody (Fig. 4B). Collectively, the results show that the AnxA2 protein was responsible for successful interactive binding with the $\mathrm{M}$ protein.

\section{Identification of the binding domains of AnxA2 that interacts with M protein in BHK-21 cells}

Since an interaction between the AnxA2 and M proteins had been detected (Fig. 4A and B), and because AnxA2 is a multi-domain protein, the AnxA2 protein was divided into five domains in order to determine the AnxA2 combination region involved in the M-AnxA2 interaction (Fig. 5A). The five domains of AnxA2 from the $\mathrm{N}$-terminal to the $\mathrm{C}$-terminal were determined, constructed, and named AnxA2- $\triangle \mathrm{I}$, AnxA2- $\Delta \mathrm{II}, \mathrm{AnxA2}-\Delta \mathrm{III}, \mathrm{AnxA2}-\Delta \mathrm{IV}$ and AnxA2- $\Delta \mathrm{V}$. The associated plasmids and vector controls were co-transfected with pcDNA3.1HA-M into BHK-21 cells for $24 \mathrm{~h}$. The Co-IP assay results showed that $M$ interacted with AnxA2- $\Delta \mathrm{I}$, AnxA2- $\Delta \mathrm{II}$, AnxA2- $\Delta \mathrm{III}$, and AnxA2- $\Delta \mathrm{IV}$, but not with AnxA2- $\Delta \mathrm{V}$, which was a deletion of a 268-334 amino acid region (Fig. 5B). These findings indicate that the AnxA2-V (268-334 aa) domain of AnxA2 is essential to the successful interaction of AnxA2 with M.

To further examine the importance of AnxA2-V in the interaction between $A n x A 2$ and $M$ proteins, the C-terminal of AnxA2 was divided into two domains (Fig. 5C). Two AnxA2 mutants were established with Flag-tag sequences (AnxA2-C1, AnxA2-C2). Two plasmids and the vector control were co-transfected with pcDNA3.1-HA-M into BHK-21 cells for $24 \mathrm{~h}$. The Co-IP assay results showed that $M$ interacted with AnxA2-C2, but not with AnxA2-C1 (Fig. 5D). Therefore, the importance of $A n x A 2-V$ in the interaction between AnxA2 and M proteins was confirmed. 
A

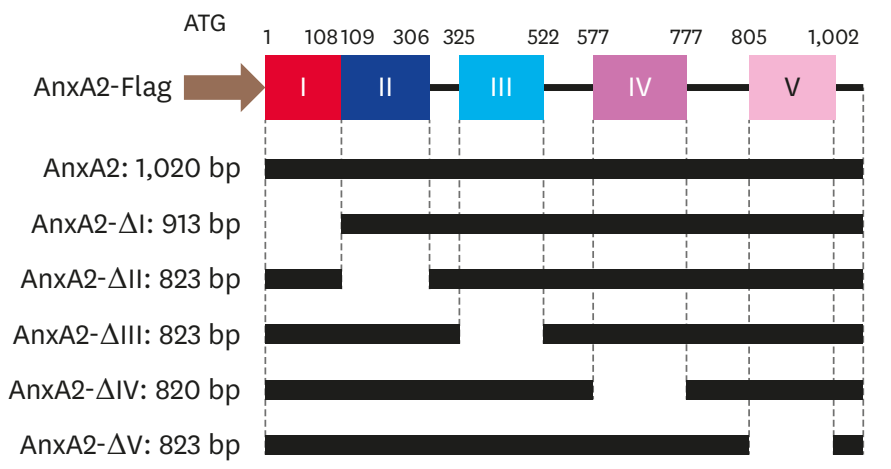

B

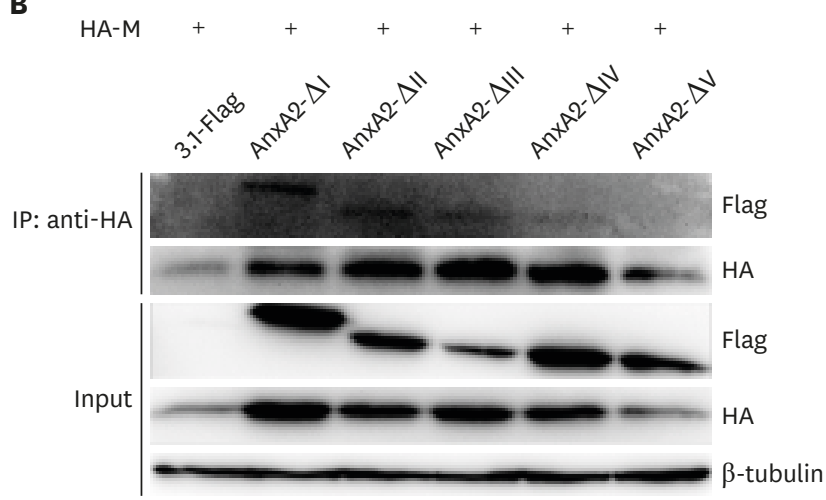

C

AnxA2-1: $450 \mathrm{bp}$

AnxA2-2: 462 bp

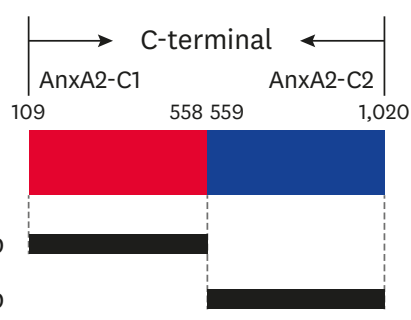

D

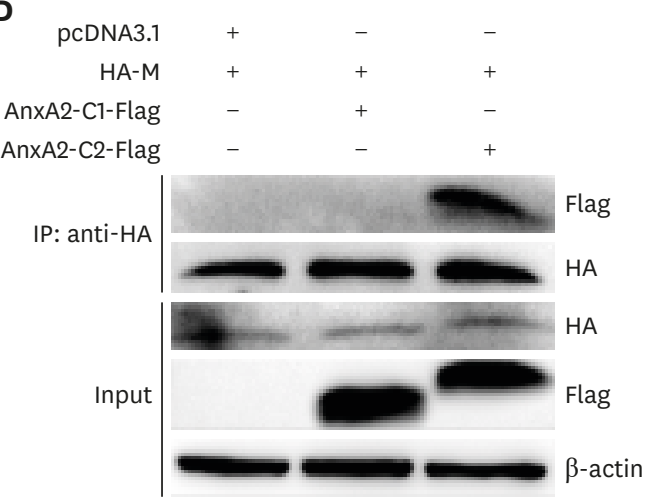

Fig. 5. Identification of the binding domains of AnXA2 that interact with $M$ in BHK-21 cells. (A) A schematic map for predicting the domains of AnxA2 protein. The full-length AnxA2 protein and five truncated AnxA2 mutants were examined. (B) Analysis of the interaction between AnxA2 and M proteins by immunoprecipitation analysis. BHK-21 cells were transfected with the pcDNA3.1-Flag vector containing AnxA2 (WT or mutant) and pcDNA3.1-HA-M. At 24 hpt, the cell supernatant was co-immunoprecipitated using anti-HA and anti-Flag antibodies. Co-immunoprecipitation of the AnxA2-M interaction was tested by western blot analysis with anti-Flag and anti-HA antibodies. Cell lysates were separated by performing $12.5 \%$ sodium dodecyl sulfate polyacrylamide gel electrophoresis and assessed by western blotting with anti-Flag and anti-HA antibodies. (C) Two truncated AnxA2 C-terminal mutants were constructed. (D) BHK-21 cells were transfected with pcDNA3.1-HA-M and AnxA2 (WT or mutant) or pcDNA3.1-Flag. Co-immunoprecipitation results for the AnxA2-M interaction were examined by western blot analysis with the anti-Flag antibody.

AnxA2, annexin A2; M, matrix; WT, wild type; hpt, hours post transfection.

\section{AnxA2 expression increases plasma membrane localization of M protein in BHK-21 cells}

To further elucidate the internal relationship between AnxA2 and M proteins, PM separation assays were performed to determine whether the level of $\mathrm{M}$ in cellular PM components would increase in the presence of AnxA2 rather than in the absence of AnxA2. BHK-21 cells were transfected with vector (pcDNA3.1), pcDNA3.1-HA-M and pcDNA3.1, or pcDNA3.1-HA-M and pcDNA3.1-AnxA2-Flag, separately, and both cytosol and PM components were collected and analyzed by western blot assays. NA/K ATPase and $\beta$-actin were used as the control compound in the PM and cytosol assays, respectively (Fig. 6). As expected, the level of M protein was reduced in cells expressing AnxA2 compared to that in cells expressing only $\mathrm{M}$ in the cytosol (Fig. 6, lanes 2 and 3). However, the levels of M in PM components were increased in cells expressing AnxA2 (Fig. 6, lanes 5 and 6) compared to that in cells that only expressed $\mathrm{M}$ in the PM. Thus, the results show that AnxA2 gene expression may affect the location of $M$ on the cell membrane. 


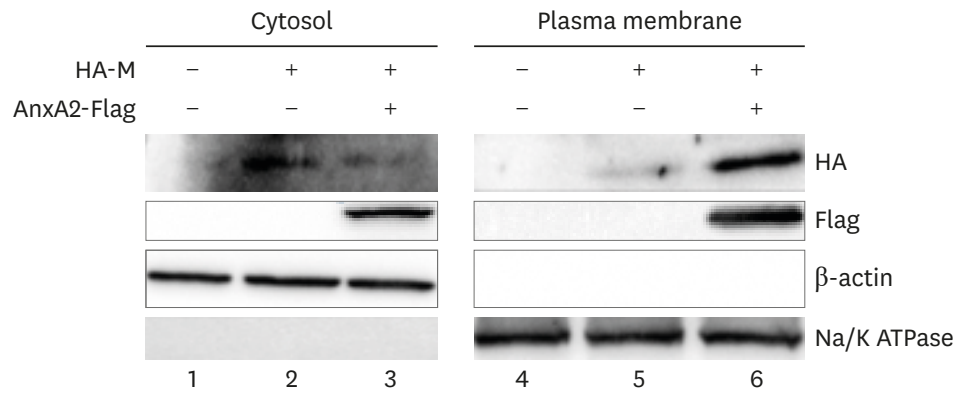

Fig. 6. AnxA2 expression increases plasma membrane localization of $M$ protein in BHK-21 cells. BHK-21 cells were transfected with vector (pcDNA3.1) alone, $M+$ vector, or $M+$ AnxA2 and at 24 hpt cells were collected, PM components separated, and western blotting performed. Na/K ATPase (Abways, China) was used as a control protein for the PM components, and $\beta$-actin (Abways) was used as a control for the cytosol fraction. AnxA2, annexin A2; M, matrix; hpt, hours post transfection; PM, plasma membrane.

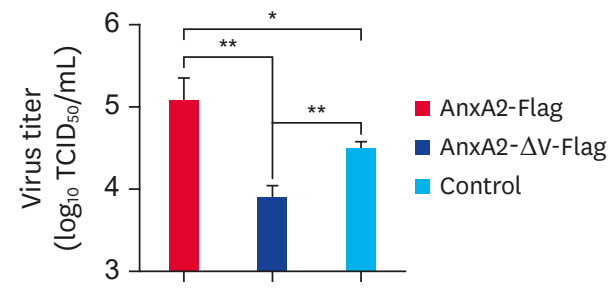

Fig. 7. The $V$ domain of the AnXA2 gene reduces the generation of BEFV progeny virus particles in BHK-21 cells. BHK-21 cells were transfected with vector control, AnXA2 + vector, or AnXA2- $\triangle \mathrm{V}+$ vector, and then infected with the virus at a multiplicity of infection of 0.1 for $48 \mathrm{~h}$. The supernatant titers of the BEFV-infected cells were determined by performing $\mathrm{TCID}_{50}$ assays.

AnXA2, annexin A2; BEFV, bovine ephemeral fever virus; $\mathrm{TCI}_{50}$, median tissue culture infective dose. ${ }^{*} p<0.05 ;{ }^{* *} p<0.01$.

\section{The $\mathrm{V}$ domain of the AnxA2 gene reduces generation of BEFV progeny virus in BHK-21 cells}

AnxA2 protein interacts with the BEFV M protein, and AnxA2 influences virus proliferation by affecting the membrane localization of $M$. To verify whether AnxA2-V affects virus replication, plasmids, including pcDNA3.1-AnxA2-Flag, pcDNA3.1-AnxA2- $\Delta \mathrm{V}$-Flag and vector control, were transfected to BHK-21 cells for $24 \mathrm{~h}$, and the cells were then infected with BEFV. Cell supernatants were collected at $48 \mathrm{hpi}$ and analysis revealed that virus titers in pcDNA3.1AnxA2- $\Delta V$-Flag cells were markedly lower than those in pcDNA3.1-AnxA2-Flag cells. In addition, compared with the vector control, virus titers in the pcDNA3.1-AnxA2- $\Delta V$-Flag cells were slightly decreased (Fig. 7). In short, AnxA2- $\Delta V$ could not promote the proliferation of $\mathrm{BEFV}$; therefore, the AnxA2-V domain of the AnxA2 gene is essential to BEFV proliferation.

\section{DISCUSSION}

Identification of host factors involved in virus infection is crucial not only to elucidate the pathogenesis of viral infections but also to develop effective antiviral strategies. However, the relationships between BEFV proliferation and host molecules have not been fully elucidated. To further investigate the replication mechanism of BEFV, we conducted a preliminary analysis of mRNA expression of BHK-21 cells infected with BEFV using high-throughput sequencing technology. The results indicated that AnxA2 gene expression increased during BEFV infection. Furthermore, the observed up-regulated expression of the AnxA2 gene was verified by performing real-time qPCR; moreover, the expression of AnxA2 protein in treated 
cells was also examined by undertaking western blot analysis. The results demonstrated that the AnxA2 gene might play a part in BEFV infection.

AnxA2 is a lipid raft-associated scaffold protein and participates in many cellular functions [40]. The AnxA2 gene has been shown to be involved in the lifecycle of several epithelial cell-targeting viruses. For instance, during virus attachment and entry, AnxA2 and A2t are central mediators of Human Papilloma Virus (HPV) entry and intracellular transport [41]. In enterovirus 71 (EV71), AnxA2 has been authenticated as a cell surface adhesion factor. AnxA2 and EV71 co-localize at the cell surface, and the AnxA2 antibody or anti-AnxA2 antibody can reduce infectivity [42]. In addition, AnxA2 or A2t have a role in viral replication [18], including that of IAV [27], porcine reproductive and respiratory syndrome virus [43], and infectious bronchitis virus [44]. Furthermore, AnxA2 may promote the production of progeny virus particles during the viral assembly and release stage. For example, in the measles virus (MV), silence of the AnxA2 gene in cervical epithelial (HeLa) cells by shRNA results in reduced production of MV progeny particles but does not influence MV entry or RNA replication $[18,40]$. In HCV, it was reported that the replication complex was reduced by knock-down of AnxA2 expression, resulting in a remarkable decrease in intracellular and extracellular virus titers $[26,45,46]$. In support of the above-mentioned observations, the present study provides further evidence that BEFV maintains and promotes AnxA2 gene expression in order to accelerate the release of progeny virus particles. Furthermore, the progeny virus titer was significantly increased in overexpressed AnxA2 gene cell lines, whereas, the titer markedly decreased when the AnxA2 gene was transiently knocked down by siRNA. Nevertheless, since endogenous AnxA2 gene expression was increased during BEFV infection at different times, there is still much research needed to further elucidate the role of the AnxA2 gene in BEFV invasion and replication.

It has been reported that the M protein of VSV can promote virus assembly and release, mainly in an endosomal sorting complex required for transport (ESCRT)-dependent way. The late domains of $\mathrm{M}$ serve to recruit the ESCRT complexes during budding, and they facilitate the release of viral particles $[8,47]$. In this study, the $\mathrm{M}$ protein promoted BEFV proliferation, and it is presumed that $M$ protein is beneficial to virus release. Nevertheless, whether the $M$ proteins of Rhabdoviridae have similar characteristics is unknown. Furthermore, it is unknown whether the conserved motif of the BEFV M protein can also facilitate the release of viral particles; thus, further experimental investigation is required.

Given that both AnxA2 and M proteins could separately promote the generation of mature virus particles, we predicted that the AnxA2 protein had an effect on the M protein, thereby mediating their interactions, which participate in the assembly and budding of BEFV. A further mechanistic study showed that AnxA2 could interact with the M protein of the BEFV in vitro, and that interaction was confirmed by the GST pull-down and Co-IP results. Subsequently, their interaction may be related to the localization of AnxA2 and M when generating mature virus particles. In this study, the PM separation assay showed that AnxA2 affected the location of $\mathrm{M}$ on the cell membrane. In the presence of AnxA2, the localization of $\mathrm{M}$ on the cell membrane increased. However, the mechanism leading to a decrease of $\mathrm{M}$ localization on the cell membrane, or determination of how AnxA2 can increase the localization of $\mathrm{M}$ on the cell membrane should be studied further. Moreover, the coimmunoprecipitation analysis further indicated that the C-terminal domain (268-334 aa) of AxnA2 contributed to this protein-protein interaction. In addition to acting as a cytoskeletaland membrane-related protein, there is growing evidence that AnxA2 may bind to viral RNA, 
which could happen in the 3' UTR of AnxA2. The presence of RNA in the immunoprecipitates of the AnxA2 separated from HeLa cells indicates that AnxA2 forms a ribonucleoprotein complex in vivo, and the $\mathrm{C}$-terminal of AnxA2 contains a key RNA-binding motif that is specific to AnxA2 [22]. It has been shown that AnxA2 combines with the framing signal in the $3^{\prime}$ non-coding region of c-myc mRNA [23,48]. In feline calicivirus (FCV), AnxA2 is associated with the genomic RNA of FCV and is necessary for efficient FCV proliferation [49]. AnxA2 and NS5B (the non-structural proteins of the hepatitis $C$ virus) interact with diverse RNAs in ternary complexes of AnxA2:NS5B:RNA, influencing the life cycle of HCV [13]. In this study, AnxA2 protein interacted with M protein, and the interaction site on AnxA2 was its C-terminal. Thus, it is suspected that the interaction between AnxA2 and M is related to RNA and that interaction affects the localization of $\mathrm{M}$ on the cell membrane. Nevertheless, the above speculations need experimental verification.

In summary, our study has demonstrated that AnxA2 gene expression is up-regulated during $\mathrm{BEFV}$ infection and that both the AnxA2 and $\mathrm{M}$ genes can promote the generation of mature virus particles. Our mechanistic study showed that the AnxA2 protein interacts with the $\mathrm{M}$ protein and mediates the position of the $\mathrm{M}$ protein at the cell membrane. Moreover, the absence of the $\mathrm{V}$ domain of the AnxA2 gene reduces the generation of BEFV progeny virus particles. These findings contribute to furthering the understanding of BEFV infection, the associated mechanisms, the search for a new potential anti-BEFV strategy.

\section{REFERENCES}

1. Walker PJ, Klement E. Epidemiology and control of bovine ephemeral fever. Vet Res (Faisalabad) 2015;46:124. PUBMED | CROSSREF

2. Hayama Y, Moriguchi S, Yanase T, Suzuki M, Niwa T, Ikemiyagi K, Nitta Y, Yamamoto T, Kobayashi S, Murai K, Tsutsui T. Epidemiological analysis of bovine ephemeral fever in 2012-2013 in the subtropical islands of Japan. BMC Vet Res 2016;12:47. PUBMED | CROSSREF

3. Du X, Zhou J. Application of biosensors to detection of epidemic diseases in animals. Res Vet Sci 2018;118:444-448. PUBMED | CROSSREF

4. Hou P, Zhao G, He C, Wang H, He H. Biopanning of polypeptides binding to bovine ephemeral fever virus $\mathrm{G}_{1}$ protein from phage display peptide library. BMC Vet Res 2018;14:3. PUBMED | CROSSREF

5. He CQ Liu YX, Wang HM, Hou PL, He HB, Ding NZ. New genetic mechanism, origin and population dynamic of bovine ephemeral fever virus. Vet Microbiol 2016;182:50-56. PUBMED | CROSSREF

6. Jayakar HR, Jeetendra E, Whitt MA. Rhabdovirus assembly and budding. Virus Res 2004;106:117-132. PUBMED | CROSSREF

7. Zan J, Liu J, Zhou JW, Wang HL, Mo KK, Yan Y, Xu YB, Liao M, Su S, Hu RL, Zhou JY. Rabies virus matrix protein induces apoptosis by targeting mitochondria. Exp Cell Res 2016;347:83-94. PUBMED | CROSSREF

8. Jayakar HR, Murti KG, Whitt MA. Mutations in the PPPY motif of vesicular stomatitis virus matrix protein reduce virus budding by inhibiting a late step in virion release. J Virol 2000;74:9818-9827. PUBMED | CROSSREF

9. Gerke V, Moss SE. Annexins: from structure to function. Physiol Rev 2002;82:331-371. PUBMED | CROSSREF

10. Mirsaeidi M, Gidfar S, Vu A, Schraufnagel D. Annexins family: insights into their functions and potential role in pathogenesis of sarcoidosis. J Transl Med 2016;14:89. PUBMED | CROSSREF 
11. Raynal P, Pollard HB. Annexins: the problem of assessing the biological role for a gene family of multifunctional calcium- and phospholipid-binding proteins. Biochim Biophys Acta 1994;1197:63-93. PUBMED | CROSSREF

12. Aliyu IA, Ling KH, Md Hashim N, Chee HY. Annexin A2 extracellular translocation and virus interaction: A potential target for antivirus-drug discovery. Rev Med Virol 2019;29:e2038. PUBMED | CROSSREF

13. Solbak SM, Abdurakhmanov E, Vedeler A, Danielson UH. Characterization of interactions between hepatitis C virus NS5B polymerase, annexin A2 and RNA - effects on NS5B catalysis and allosteric inhibition. Virol J 2017;14:236. PUBMED | CROSSREF

14. Woodham AW, Taylor JR, Jimenez AI, Skeate JG, Schmidt T, Brand HE, Da Silva DM, Kast WM. Small molecule inhibitors of the annexin A2 heterotetramer prevent human papillomavirus type 16 infection. J Antimicrob Chemother 2015;70:1686-1690. PUBMED | CROSSREF

15. Wang J, Song J, Clark G, Roux SJ. ANN1 and ANN2 function in post-phloem sugar transport in root tips to affect primary root growth. Plant Physiol 2018;178:390-401. PUBMED | CROSSREF

16. Luo S, Xie C, Wu P, He J, Tang Y, Xu J, Zhao S. Annexin A2 is an independent prognostic biomarker for evaluating the malignant progression of laryngeal cancer. Exp Ther Med 2017;14:6113-6118. PUBMED | CROSSREF

17. Shaker MK, Abdel Fattah HI, Sabbour GS, Montasser IF, Abdelhakam SM, El Hadidy E, Yousry R, El Dorry AK. Annexin A2 as a biomarker for hepatocellular carcinoma in Egyptian patients. World J Hepatol 2017;9:469-476. PUBMED | CROSSREF

18. Taylor JR, Skeate JG, Kast WM. Annexin A2 in virus infection. Front Microbiol 2018;9:2954. PUBMED | CROSSREF

19. Nazmi AR, Ozorowski G, Pejic M, Whitelegge JP, Gerke V, Luecke H. N-terminal acetylation of annexin A2 is required for S100A10 binding. Biol Chem 2012;393:1141-1150. PUBMED | CROSSREF

20. Woodham AW, Da Silva DM, Skeate JG, Raff AB, Ambroso MR, Brand HE, Isas JM, Langen R, Kast WM. The S100A10 subunit of the annexin A2 heterotetramer facilitates L2-mediated human papillomavirus infection. PLoS One 2012;7:e43519. PUBMED | CROSSREF

21. Grindheim AK, Saraste J, Vedeler A. Protein phosphorylation and its role in the regulation of Annexin A2 function. Biochim Biophys Acta, Gen Subj 2017;1861:2515-2529. PUBMED | CROSSREF

22. Filipenko NR, MacLeod TJ, Yoon CS, Waisman DM. Annexin A2 is a novel RNA-binding protein. J Biol Chem 2004;279:8723-8731. PUBMED | CROSSREF

23. Mickleburgh I, Burtle B, Hollås H, Campbell G, Chrzanowska-Lightowlers Z, Vedeler A, Hesketh J. Annexin A2 binds to the localization signal in the $3^{\prime}$ untranslated region of c-myc mRNA. FEBS J 2005;272:413-421. PUBMED | CROSSREF

24. Wright JF, Kurosky A, Wasi S. An endothelial cell-surface form of annexin II binds human cytomegalovirus. Biochem Biophys Res Commun 1994;198:983-989. PUBMED | CROSSREF

25. Wright JF, Kurosky A, Pryzdial EL, Wasi S. Host cellular annexin II is associated with cytomegalovirus particles isolated from cultured human fibroblasts. J Virol 1995;69:4784-4791. PUBMED | CROSSREF

26. Backes P, Quinkert D, Reiss S, Binder M, Zayas M, Rescher U, Gerke V, Bartenschlager R, Lohmann V. Role of annexin A2 in the production of infectious hepatitis C virus particles. J Virol 2010;84:5775-5789. PUBMED | CROSSREF

27. LeBouder F, Morello E, Rimmelzwaan GF, Bosse F, Péchoux C, Delmas B, Riteau B. Annexin II incorporated into influenza virus particles supports virus replication by converting plasminogen into plasmin. J Virol 2008;82:6820-6828. PUBMED | CROSSREF

28. Ma Y, Sun J, Gu L, Bao H, Zhao Y, Shi L, Yao W, Tian G, Wang X, Chen H. Annexin A2 (ANXA2) interacts with nonstructural protein 1 and promotes the replication of highly pathogenic $\mathrm{H} 5 \mathrm{~N} 1$ avian influenza virus. BMC Microbiol 2017;17:191. PUBMED | CROSSREF 
29. Liu Y, Gao P. Modulation of hepatitis B surface antigen secretion by annexin II expressed in hepatitis B virus-producing hepatoma cells. Mol Med Rep 2014;10:3113-3117. PUBMED | CROSSREF

30. Sheng C, Liu X, Jiang Q, Xu B, Zhou C, Wang Y, Chen J, Xiao M. Annexin A2 is involved in the production of classical swine fever virus infectious particles. J Gen Virol 2015;96:1027-1032. PUBMED | CROSSREF

31. Hou P, Wang H, Zhao G, He C, He H. Rapid detection of infectious bovine Rhinotracheitis virus using recombinase polymerase amplification assays. BMC Vet Res 2017;13:386. PUBMED | CROSSREF

32. Wang X, Ju Z, Huang J, Hou M, Zhou L, Qi C, Zhang Y, Gao Q, Pan Q, Li G, Zhong J, Wang C. The relationship between the variants of the bovine MBL2 gene and milk production traits, mastitis, serum MBL-C levels and complement activity. Vet Immunol Immunopathol 2012;148:311-319. PUBMED | CROSSREF

33. Chang XB, Yang YQ, Gao JC, Zhao K, Guo JC, Ye C, Jiang CG, Tian ZJ, Cai XH, Tong GZ, An TQ. Annexin $\mathrm{A} 2$ binds to vimentin and contributes to porcine reproductive and respiratory syndrome virus multiplication. Vet Res (Faisalabad) 2018;49:75. PUBMED | CROSSREF

34. Wang XG, Huang JM, Feng MY, Ju ZH, Wang CF, Yang GW, Yuan JD, Zhong JF. Regulatory mutations in the A2M gene are involved in the mastitis susceptibility in dairy cows. Anim Genet 2014;45:28-37. PUBMED | CROSSREF

35. Li S, van Os GM, Ren S, Yu D, Ketelaar T, Emons AM, Liu CM. Expression and functional analyses of EXO70 genes in Arabidopsis implicate their roles in regulating cell type-specific exocytosis. Plant Physiol 2010;154:1819-1830. PUBMED | CROSSREF

36. Lv L, Zhao G, Wang H, He H. Cholesterol 25-Hydroxylase inhibits bovine parainfluenza virus type 3 replication through enzyme activity-dependent and -independent ways. Vet Microbiol 2019;239:108456. PUBMED | CROSSREF

37. Hou P, Zhao M, He W, He H, Wang H. Cellular microRNA bta-miR-2361 inhibits bovine herpesvirus 1 replication by directly targeting EGR1 gene. Vet Microbiol 2019;233:174-183. PUBMED | CROSSREF

38. Ma W, Wang H, He H. Bovine herpesvirus 1 tegument protein UL41 suppresses antiviral innate immune response via directly targeting STAT1. Vet Microbiol 2019;239:108494. PUBMED | CROSSREF

39. Liang J, Sagum CA, Bedford MT, Sidhu SS, Sudol M, Han Z, Harty RN. Chaperone-mediated autophagy protein BAG3 negatively regulates ebola and marburg VP40-mediated egress. PLoS Pathog 2017;13:e1006132. PUBMED | CROSSREF

40. Koga R, Kubota M, Hashiguchi T, Yanagi Y, Ohno S. Annexin A2 mediates the localization of measles virus matrix protein at the plasma membrane. J Virol 2018;92:92. PUBMED | CROSSREF

41. Dziduszko A, Ozbun MA. Annexin A2 and S100A10 regulate human papillomavirus type 16 entry and intracellular trafficking in human keratinocytes. J Virol 2013;87:7502-7515. PUBMED | CROSSREF

42. Yang SL, Chou YT, Wu CN, Ho MS. Annexin II binds to capsid protein VP1 of enterovirus 71 and enhances viral infectivity. J Virol 2011;85:11809-11820. PUBMED | CROSSREF

43. Li J, Guo D, Huang L, Yin M, Liu Q, Wang Y, Yang C, Liu Y, Zhang L, Tian Z, Cai X, Yu L, Weng C. The interaction between host Annexin A2 and viral Nsp9 is beneficial for replication of porcine reproductive and respiratory syndrome virus. Virus Res 2014;189:106-113. PUBMED | CROSSREF

44. Kwak H, Park MW, Jeong S. Annexin A2 binds RNA and reduces the frameshifting efficiency of infectious bronchitis virus. PLoS One 2011;6:e24067. PUBMED | CROSSREF

45. Lai CK, Jeng KS, Machida K, Lai MM. Association of hepatitis $C$ virus replication complexes with microtubules and actin filaments is dependent on the interaction of NS3 and NS5A. J Virol 2008;82:8838-8848. PUBMED | CROSSREF

46. Saxena V, Lai CK, Chao TC, Jeng KS, Lai MM. Annexin A2 is involved in the formation of hepatitis C virus replication complex on the lipid raft. J Virol 2012;86:4139-4150.

PUBMED | CROSSREF 
47. Soh TK, Whelan SP. Tracking the fate of genetically distinct vesicular stomatitis virus matrix proteins highlights the role for late domains in assembly. J Virol 2015;89:11750-11760. PUBMED | CROSSREF

48. Hollås H, Aukrust I, Grimmer S, Strand E, Flatmark T, Vedeler A. Annexin A2 recognises a specific region in the 3'-UTR of its cognate messenger RNA. Biochim Biophys Acta 2006;1763:1325-1334. PUBMED | CROSSREF

49. Santos-Valencia JC, Cancio-Lonches C, Trujillo-Uscanga A, Alvarado-Hernández B, Lagunes-Guillén A, Gutiérrez-Escolano AL. Annexin A2 associates to feline calicivirus RNA in the replication complexes from infected cells and participates in an efficient viral replication. Virus Res 2019;261:1-8. PUBMED | CROSSREF 